\title{
THE BIFURCATION SET OF THE PERIOD FUNCTION OF THE DEHOMOGENIZED LOUD'S CENTERS IS BOUNDED
}

\author{
F. MAÑOSAS AND J. VILLADELPRAT \\ (Communicated by Carmen C. Chicone)
}

\begin{abstract}
This paper is concerned with the behaviour of the period function of the quadratic reversible centers. In this context the interesting stratum is the family of the so-called Loud's dehomogenized systems, namely

$$
\left\{\begin{array}{l}
\dot{x}=-y+x y \\
\dot{y}=x+D x^{2}+F y^{2} .
\end{array}\right.
$$

In this paper we show that the bifurcation set of the period function of these centers is contained in the rectangle $K=(-7,2) \times(0,4)$. More concretely, we prove that if $(D, F) \notin K$, then the period function of the center is monotonically increasing.
\end{abstract}

\section{IntroduCtion AND STATEMENT OF the RESUlt}

This paper is devoted to studying the period function of the quadratic centers, and it constitutes the continuation of the results obtained in [14]. There are four families of quadratic centers: Hamiltonian, reversible, codimension four and generalized Lotka-Volterra systems. Chicone has conjectured [2] that the reversible centers have at most two critical periods and that the centers of the three other families have a monotonic period function. The behaviour of the period function of the quadratic centers has been studied extensively, and there is much analytic evidence that the conjecture is true (see $[4,7,9,11,13,15,16]$ and the references therein). It is clear therefore that in this setting the most interesting family of centers is the reversible one. It is well known that any reversible quadratic center can be brought to Loud's normal form

$$
\left\{\begin{array}{l}
\dot{x}=-y+B x y \\
\dot{y}=x+D x^{2}+F y^{2}
\end{array}\right.
$$

by means of an affine transformation and a constant rescaling of time. It is proved in [5] that if $B=0$, then the period function of the center is monotonically increasing. The remaining cases, namely $B \neq 0$, can be brought with a rescaling to $B=1$, i.e.,

$$
\left\{\begin{array}{l}
\dot{x}=-y+x y \\
\dot{y}=x+D x^{2}+F y^{2}
\end{array}\right.
$$

Received by the editors October 18, 2006.

2000 Mathematics Subject Classification. Primary 34C07, 34C23; Secondary 34C25.

The authors were partially supported by the CONACIT through the grant 2005-SGR-00550 and by the DGES through the grant MTM-2005-06098-C02-1. 
This is precisely the family of the so-called dehomogenized Loud's systems, and our goal in this paper is to prove the following result:

Theorem A. If $(D, F) \notin(-7,2) \times(0,4)$, then the center at the origin of system (1) has a monotonically increasing period function.

Theorem A implies that the bifurcation set of the period function of the family of centers (1) is contained in the rectangle $K=(-7,2) \times(0,4)$. This bifurcation diagram has been studied in the series of papers $[6,7,8]$ and the results that we obtain here agree with the conjectural bifurcation diagram presented in [7].

The proof of Theorem A follows from the combination of the result in [14] with three propositions that we prove in Section 3. As a matter of fact these propositions provide a parameter subset $K$ that is smaller than the rectangle $(-7,2) \times(0,4)$. However, since this "optimal" $K$ is involved, to describe the result, for the sake of simplicity, we prefer to state the result with $K=(-7,2) \times(0,4)$.

Let us conclude this section by quoting other results pertaining to the period function of the dehomogenized Loud's centers. By using a Picard-Fuchs approach, Yulin Zhao $[17,18]$ describes completely the behaviour of the period function in $F=3 / 2$ and $F=2$. Chouikha [3] shows the monotonicity in the straight lines $F+2 D=1$ and $F=-1$ and some segments inside $D=-1 / 2, D=0, F=1$ and $F=2$. Finally, we also refer to the work of Changjian Liu [1]. By using the results in [14] he shows that there exists some bounded set $K$ such that the period function is monotonically increasing for $\mu \notin K$. This result is similar to Theorem A, but his approach does not allow us to give $K$ explicitly.

\section{Previous Results}

The proof of Theorem A relies strongly on the results in [14]. The main result in that paper is Theorem 2.1 below and in its statement, see Figure $1, M_{i}$ stands for the union of the light grey regions and $M_{d}$ is the rectangle $\left(-1,-\frac{1}{2}\right) \times\left(\frac{4}{3}, 2\right)$ in dark grey.

Theorem 2.1. The period function of the center at the origin of system (1) is monotonically increasing (respectively, decreasing) in the case that $\mu \in M_{i}$ (respectively, $\mu \in M_{d}$ ).

Here and in what follows we use the notation $\mu:=(D, F)$. Our goal is to find a bounded set $K$ in the parameter plane such that if $\mu \notin K$, then the center of system (1) has a monotonically increasing period function. To this end it is necessary to study the unbounded regions not covered by Theorem 2.1, namely the straight lines $F D(F-2)(D+1)=0$ and two horizontal strips. The boundary of the right strip leans on $F(F-1)=0$, while the boundary of the left strip leans on $R(\mu)=0$, where

$$
\begin{aligned}
R(\mu)= & -\left(745 F^{4}-2800 F^{3}+2688 F^{2}-442 F+52\right)(2 D+1)^{4} \\
& -3 F(F-2)\left(125 F^{4}-518 F^{3}+174 F^{2}+382 F-1\right)(2 D+1)^{2} \\
& +9 F^{2}(3 F-4)(F-2)^{2}(F+2)^{3} .
\end{aligned}
$$

In order to prove Theorem A we shall first show that there exists a coordinate transformation that brings (1) to a potential system. Recall that if $V$ is a smooth 


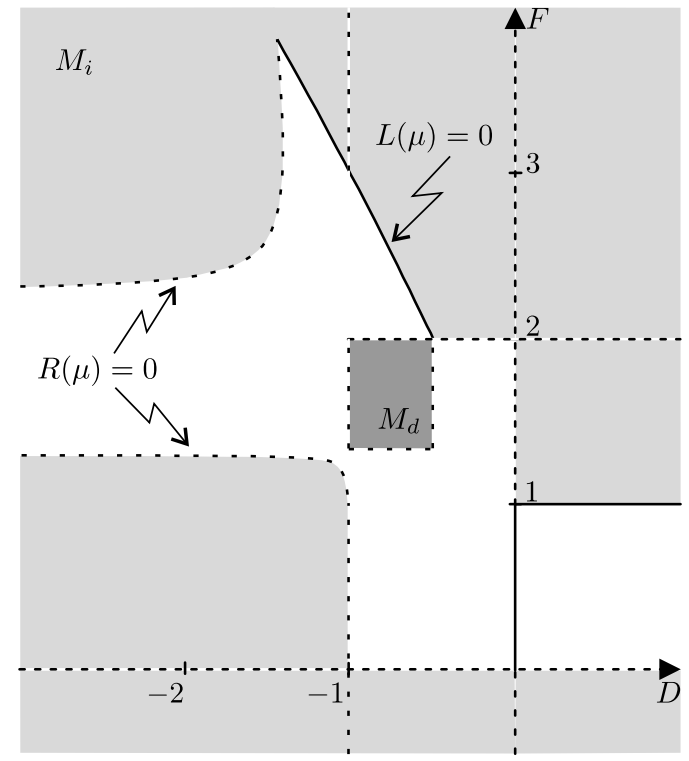

Figure 1. Monotonicity regions according to the results in [14].

function with $V^{\prime}(0)=0$ and $V^{\prime \prime}(0)>0$, then the potential system

$$
\left\{\begin{array}{l}
\dot{u}=-v \\
\dot{v}=V^{\prime}(u)
\end{array}\right.
$$

has a center at the origin. For these centers we have the following monotonicity criterium due to $R$. Schaaf [12]. In its statement $\mathcal{I}$ denotes the projection on the $u$-axis of the period annulus of the center.

Theorem 2.2 (Schaaf's criterium). The center at the origin of system (3) has a monotonically increasing period function in the case that

$\left(I_{1}\right) \quad 5 V^{\prime \prime \prime}(u)^{2}-3 V^{\prime \prime}(u) V^{(4)}(u)>0$ for any $u \in \mathcal{I}$ with $V^{\prime \prime}(u)>0$

and

$\left(I_{2}\right) \quad V^{\prime}(u) V^{\prime \prime \prime}(u)<0$ for any $u \in \mathcal{I}$ with $V^{\prime \prime}(u)=0$.

On the other hand, in the case that

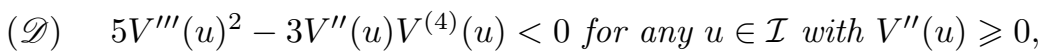

then the period function is monotonically decreasing.

The next result provides the coordinate transformation that we mention above. The proof is omitted since it is a straightforward computation.

Lemma 2.3. If $F \neq 0$, then the transformation $\left\{u=\frac{(1-x)^{-F}-1}{F}, v=(1-x)^{-F} y\right\}$ brings system (1) to

$$
\left\{\begin{array}{l}
\dot{u}=-v \\
\dot{v}=(F u+1)\left(1-(F u+1)^{\frac{-1}{F}}\right)\left(D+1-D(F u+1)^{-\frac{1}{F}}\right) .
\end{array}\right.
$$


If $F=0$, then the coordinate transformation $\{u=-\ln (1-x), v=y\}$ brings (1) to

$$
\left\{\begin{array}{l}
\dot{u}=-v, \\
\dot{v}=\left(1-e^{-u}\right)\left(D+1-D e^{-u}\right) .
\end{array}\right.
$$

Note that (4) and (5) are potential systems. For instance if $F \notin\left\{0, \frac{1}{2}, 1\right\}$, then the associated potential is

$$
V(u)=(F u+1)^{2} q\left((F u+1)^{\frac{-1}{F}}\right)+\frac{F-D-1}{2 F(2 F-1)(F-1)},
$$

where we take

$$
q(z):=\frac{D+1}{2 F}-\frac{2 D+1}{2 F-1} z+\frac{D}{2(F-1)} z^{2} .
$$

In addition, one can check that if $F \neq 0$, then

$$
\begin{aligned}
& V^{\prime \prime}(u)=-(2 D+1)(F-1)(F u+1)^{\frac{-1}{F}}+D(F-2)(F u+1)^{\frac{-2}{F}}+F(D+1), \\
& V^{\prime \prime \prime}(u)=(2 D+1)(F-1)(F u+1)^{\frac{-1}{F}-1}-2 D(F-2)(F u+1)^{\frac{-2}{F}-1}, \\
& V^{(4)}(u)=-(2 D+1)\left(F^{2}-1\right)(F u+1)^{\frac{-1}{F}}-2+2 D\left(F^{2}-4\right)(F u+1)^{\frac{-2}{F}-2} .
\end{aligned}
$$

Remark 2.4. Let us introduce the auxiliary function $g(u):=(F u+1)^{\frac{-1}{F}}$. Then, setting $z=g(u)$, from the above equalities it follows that

$$
V^{\prime}(u)=(F u+1) V_{1}(z), V^{\prime \prime}(u)=V_{2}(z), V^{\prime \prime \prime}(u)=\frac{V_{3}(z)}{F u+1}, V^{(4)}(u)=\frac{V_{4}(z)}{(F u+1)^{2}},
$$

where each $V_{i}$ is a polynomial. Taking this into account it turns out that

$$
5 V^{\prime \prime \prime}(u)^{2}-3 V^{\prime \prime}(u) V^{(4)}(u)=\frac{5 V_{3}(z)^{2}-3 V_{2}(z) V_{4}(z)}{(F u+1)^{2}},
$$

and we observe that the numerator of the last expression is polynomial in $z$ as well. More concretely one can check that $5 V_{3}(z)^{2}-3 V_{2}(z) V_{4}(z)=z S(z)$, where

$$
\begin{aligned}
S(z):= & -2 D^{2}(3 F-4)(F-2)^{2} z^{3}+D(9 F-5)(F-1)(F-2)(2 D+1) z^{2} \\
& +\frac{1}{2}\left(\left(F^{2}(16-9 F)-2 F+4\right)(2 D+1)^{2}\right. \\
& \left.+3 F\left(F^{2}-4\right)\right) z+3 F(D+1)(2 D+1)\left(F^{2}-1\right) .
\end{aligned}
$$

This fact simplifies the application of Schaaf's criterium because it allows us to verify its conditions by using polynomial functions in $z$. Indeed, due to $z=g(u)>0$, condition $I_{1}$ is equivalent to showing that $S(z)>0$ for any $z \in g(\mathcal{I})$ with $V_{2}(z)>0$. Concerning condition $I_{2}$ let us point out that $V^{\prime}(u) V^{\prime \prime \prime}(u)=V_{1}(z) V_{3}(z)$.

\section{Proof of the RESUlT}

This section is devoted to proving Theorem A. To this end note that Lemma 2.3 enables us to consider a center of a potential system instead of the original dehomogenized Loud's center. We can thus apply Schaaf's criterium to study the monotonicity of its period function. Then the proof is organized as follows. Proposition 3.1 deals with the parameters $\mu=(D, F)$ in the right strip, and it shows that if $F>2$, then the center has a monotonically increasing period function. Proposition 3.2 shows that this is also the case for those parameters inside the left strip with $F<-7$. Finally the parameters inside the straight lines $F D(F-2)(D+1)=0$ are 


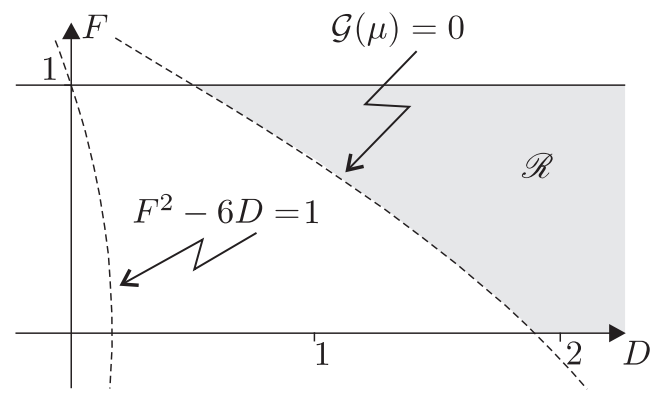

FiguRE 2. The subset $\mathscr{R}$ in the right strip.

studied in Proposition 3.6. The combination of these propositions with Theorem 2.1 completes the proof of Theorem A.

3.1. Monotonicity in the right strip. Define $\mathcal{G}(\mu):=6 D^{2}+\left(2 F^{2}+6 F-5\right) D-$ $(F-4)\left(F^{2}+F-3\right)$. The curve $\mathcal{G}(\mu)=0$ splits the strip $(0,+\infty) \times(0,1)$ into two connected components (see Figure 2 ). In the statement of the next result, $\mathscr{R}$ stands for the unbounded component.

Proposition 3.1. If $\mu \in \mathscr{R}$, then the period function of (1) is increasing.

Proof. Let $\mathcal{I}$ be the projection on the $u$-axis of the period annulus of the center of system (4). It is easy to show that for the parameter values under consideration $V$ is increasing for $u>0$ and that it has a local maximum at $u^{\star}:=\frac{(1+1 / D)^{-F}-1}{F}$. Thus the left endpoint of $\mathcal{I}$ is precisely $u^{\star}$ and accordingly $V(u)<V\left(u^{\star}\right)$ for all $u \in \mathcal{I}$. By Lemma 2.4 in [14], system (4) satisfies condition $I_{2}$ of Schaaf's criterium for all $\mu \in(0,+\infty) \times(0,1)$. We claim that if $\mu \in \mathscr{R}$, then

$$
V^{(4)}(u)<0 \text { for all } u \in \mathcal{I},
$$

which clearly implies condition $I_{1}$ of Schaaf's criterium. Recall that

$$
V^{(4)}(u)=(F u+1)^{\frac{-1}{F}-2}\left(2 D\left(F^{2}-4\right)(F u+1)^{\frac{-1}{F}}-(2 D+1)\left(F^{2}-1\right)\right) .
$$

Hence $V^{(4)}$ changes its signum only once, at $u=u_{0}$, characterized by

$$
\left(F u_{0}+1\right)^{\frac{-1}{F}}=\frac{(2 D+1)\left(F^{2}-1\right)}{2 D\left(F^{2}-4\right)}=: s_{0} .
$$

Since $V^{(4)}(0)=1-6 D-F^{2}$ is negative for $\mu \in \mathscr{R}$ (see Figure 2), to prove the claim it suffices to show that $V\left(u_{0}\right)>V\left(u^{\star}\right)$ because then $u_{0} \notin \mathcal{I}$.

Assume $F \neq 1 / 2$ first. Then, from (6), the inequality $V\left(u_{0}\right)>V\left(u^{\star}\right)$ can be written as

$$
\left(s_{0}\right)^{-2 F} q\left(s_{0}\right)>(1+1 / D)^{-2 F} q(1+1 / D) .
$$


Here we took $\left(F u^{\star}+1\right)^{\frac{-1}{F}}=1+1 / D$ and $\left(F u_{0}+1\right)^{\frac{-1}{F}}=s_{0}$ into account. Since

$$
q(1+1 / D)=\frac{(D+1)(D+F)}{2 D F(F-1)(2 F-1)}
$$

is negative for $\mu \in(0,+\infty) \times\left(\frac{1}{2}, 1\right)$ and positive for $\mu \in(0,+\infty) \times\left(0, \frac{1}{2}\right)$, the above inequality splits into

$$
\left(\frac{D s_{0}}{D+1}\right)^{2 F}<\frac{q\left(s_{0}\right)}{q(1+1 / D)} \text { if } F<1 / 2
$$

and

$$
\left(\frac{D s_{0}}{D+1}\right)^{2 F}>\frac{q\left(s_{0}\right)}{q(1+1 / D)} \text { if } F>1 / 2
$$

To prove these inequalities we shall use the third degree Taylor's polynomial of $x \longmapsto(1-x)^{2 F}$ at $x=0$. We have that $(1-x)^{2 F}=P_{3}(x)+R(x)$ where

$$
P_{3}(x)=1-2 F x+F(2 F-1) x^{2}-\frac{2}{3} F(2 F-1)(F-1) x^{3}
$$

and

$$
R(x)=\frac{1}{6} F(F-1)(2 F-1)(2 F-3)(1-\xi)^{2 F-4} x^{4}
$$

for some $\xi \in\langle 0, x\rangle$. Clearly $(1-x)^{2 F}-P_{3}(x)=R(x)$ is positive for $F \in\left(\frac{1}{2}, 1\right)$ and negative for $F \in\left(0, \frac{1}{2}\right)$. Taking this into account, to prove (8) and (9) it suffices to verify that

$$
P_{3}\left(1-\frac{D s_{0}}{D+1}\right)-\frac{q\left(s_{0}\right)}{q(1+1 / D)}
$$

is positive for $\mu \in(0,+\infty) \times\left(\frac{1}{2}, 1\right)$ and negative for $\mu \in(0,+\infty) \times\left(0, \frac{1}{2}\right)$. Some computations yield

$$
P_{3}\left(1-\frac{D s_{0}}{D+1}\right)-\frac{q\left(s_{0}\right)}{q(1+1 / D)}=\frac{F(F-1)(2 F-1)\left(F^{2}-6 D-7\right)^{2} \mathcal{G}(\mu)}{12\left(F^{2}-4\right)^{3}(D+F)(D+1)^{3}},
$$

where

$$
\mathcal{G}(\mu)=6 D^{2}+\left(2 F^{2}+6 F-5\right) D-(F-4)\left(F^{2}+F-3\right) .
$$

Since the curve $\mathcal{G}(\mu)=0$ intersects the strip $(0,+\infty) \times(0,1)$ as shown in Figure 2, the above expression proves the validity of the inequalities in (8) and (9) for $\mu \in \mathscr{R}$. Accordingly the claim is true for $F \neq 1 / 2$. The case $F=1 / 2$ follows easily by continuity and its proof is omitted here for the sake of brevity.

In short, conditions $I_{1}$ and $I_{2}$ of Schaaf's criterium hold, and so we can assert that the period function is monotonically increasing for $\mu \in \mathscr{R}$. 


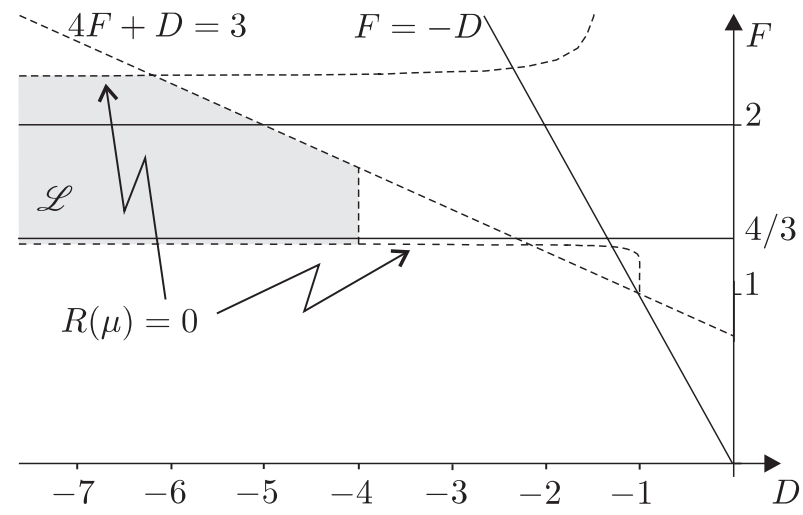

Figure 3 . The subset $\mathscr{L}$ in the left strip.

3.2. Monotonicity in the left strip. This section is devoted to studying the parameters inside the left strip, and we prove the following result, which refers to the light grey subset $\mathscr{L}$ in Figure 3 .

Proposition 3.2. If $\mu \in \mathscr{L}$, then the period function of (1) is increasing.

To prove this proposition we need some auxiliary lemmas. The first one is concerned with the projection of the period annulus on the $u$-axis. The location of this interval will be very important in order to apply Shaaf's monotonicity criterium.

Lemma 3.3. Let $\mathcal{I}$ be the projection on the u-axis of the period annulus of the center at the origin of (4). If $\mu \in \mathscr{L}$, then

$$
\mathcal{I} \subset\left(\frac{(1-1 / D)^{-F}-1}{F}, \frac{(1+1 / D)^{-F}-1}{F}\right) .
$$

More concretely, the right endpoint of $\mathcal{I}$ is $\frac{(1+1 / D)^{-F}-1}{F}$ and the left endpoint is greater than $\frac{(1-1 / D)^{-F}-1}{F}$.

Proof. Note, see Figure 3, that $\mathscr{L} \subset \mathscr{U}:=\left\{\mu \in \mathbb{R}^{2}: 1<F<-D\right\}$. One can easily verify that if $\mu \in \mathscr{U}$, then $V$ is decreasing for $u<0$ and that it has a local maximum at $u^{\star}:=\frac{(1+1 / D)^{-F}-1}{F}$. Hence the right endpoint of $\mathcal{I}$ is $u^{\star}$ and, consequently, $V(u)<V\left(u^{\star}\right)$ for $u \in \mathcal{I}$. Therefore, to prove the result it suffices to check that $u_{0}:=\frac{(1-1 / D)^{-F}-1}{F}$ is negative and $V\left(u_{0}\right)>V\left(u^{\star}\right)$. This inequality, on account of (6), can be written as

$$
(1-1 / D)^{-2 F} q(1-1 / D)>(1+1 / D)^{-2 F} q(1+1 / D) .
$$

Since $q(1+1 / D)=\frac{(D+1)(D+F)}{2 D F(F-1)(2 F-1)}$ is negative for $\mu \in \mathscr{U}$, this is equivalent to

$$
\left(1-\frac{2}{D+1}\right)^{2 F}>\frac{q(1-1 / D)}{q(1+1 / D)} .
$$

To study this inequality we use the Taylor development of $x \longmapsto(1-x)^{2 F}$ at $x=0$. For the sake of convenience we consider two cases: $F \geqslant 3 / 2$ and $F<3 / 2$.

Assume $F \geqslant 3 / 2$ first. Then we have that $(1-x)^{2 F} \geqslant P_{3}(x)$, where

$$
P_{3}(x):=1-2 F x+F(2 F-1) x^{2}-\frac{2}{3} F(2 F-1)(F-1) x^{3} .
$$




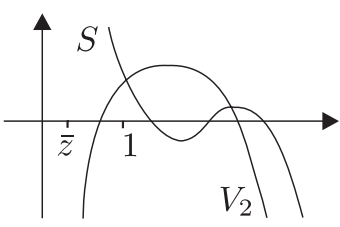

$\mu \in \mathscr{L}_{1}$

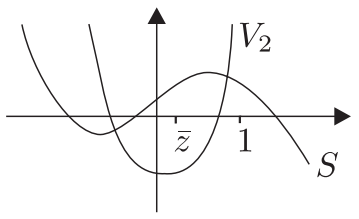

$\mu \in \mathscr{L}_{2}$

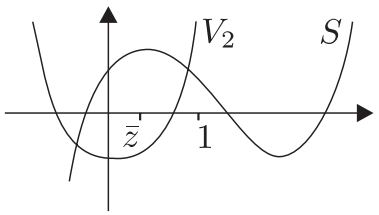

$\mu \in \mathscr{L}_{3}$

Figure 4 . Location of $\bar{z}=1+\frac{1}{D}$ and $z=1$.

Indeed, this is so because by Taylor's Theorem we can assert that there exists $\xi \in\langle 0, x\rangle$ so that $(1-x)^{2 F}-P_{3}(x)=\frac{1}{6} F(F-1)(2 F-1)(2 F-3)(1-\xi)^{2 F-4} x^{4}$, which is positive. Hence $\left(1-\frac{2}{D+1}\right)^{2 F} \geqslant P_{3}\left(\frac{2}{D+1}\right)$ and so in order to prove $(10)$ it suffices to show that

$$
P_{3}\left(\frac{2}{D+1}\right)-\frac{q(1-1 / D)}{q(1+1 / D)}=-\frac{4}{3} \frac{F(F-1)(2 F-1)(4 F+D-3)}{(D+1)^{3}(D+F)}
$$

is positive for $\mu \in \mathscr{L}$. In view of Figure 3 , the fact that it is different from zero for all $\mu \in \mathscr{L}$ is clear. Taking this into account, we conclude that it is positive by evaluating the expression in a concrete parameter. The case $F \in(1,3 / 2)$ follows exactly the same way but using the Taylor polynomial of fourth degree. We do not include it for the sake of brevity.

In short we have shown that $u_{0} \notin \mathcal{I}$ for $\mu \in \mathscr{L}$. Since one can easily see that $u_{0}<0$ for these parameter values, this implies that $u_{0}$ is smaller than the left endpoint of $\mathcal{I}$, as desired.

Let us define at this point the subsets of the parameter plane given by

$$
\mathscr{L}_{1}=\{\mu \in \mathscr{L}: F>2\}, \mathscr{L}_{2}=\left\{\mu \in \mathscr{L}: F \in\left(\frac{4}{3}, 2\right)\right\} \text { and } \mathscr{L}_{3}=\left\{\mu \in \mathscr{L}: F<\frac{4}{3}\right\} .
$$

The next result refers to the polynomials $S$ and $V_{2}$ in Remark 2.4. Recall that by means of $z=g(u):=(F u+1)^{\frac{-1}{F}}$, these polynomials give information concerning the functions that appear in Schaaf's criterium. Notice in particular that $g(0)=1$ and $g\left(\frac{(1 \pm 1 / D)^{-F}-1}{F}\right)=1 \pm \frac{1}{D}$.

Lemma 3.4. If $\mu \in \mathscr{L}_{1} \cup \mathscr{L}_{2} \cup \mathscr{L}_{3}$, then the relative position of $z=1+\frac{1}{D}$ and $z=1$ with respect to the zeros of $S$ and $V_{2}$ is as shown in Figure 4.

Proof. The distribution of the roots of $V_{2}$ and $S$ and their location with respect to $z=0$ was obtained in [14]. Therefore in order to prove the result we must only "place" $z=1$ and $z=1+\frac{1}{D}$ in the graphics. In the proof we shall use that

$$
V_{2}(1)=1, V_{2}^{\prime}(1)=1-2 D-F, V_{2}(1+1 / D)=-(1+1 / D),
$$

and

$$
\begin{aligned}
& S(1)=2\left(10 D^{2}+10 D F-D+4 F^{2}-5 F+1\right), \\
& S^{\prime}(1)=8(8-5 F) D^{2}-\left(32 F^{2}-62 F+12\right) D+(2-3 F)(F-1)^{2}, \\
& S^{\prime \prime}(1)=2 D(F-2)(2(16 F-19) D+(9 F-5)(F-1)) .
\end{aligned}
$$




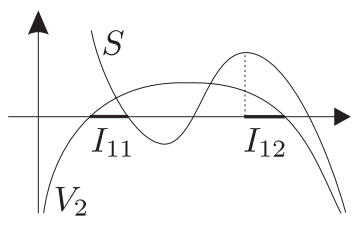

Figure 5. Intervals where $z=1$ may be located in the case that $\mu \in \mathscr{L}_{1}$.

Thus, for $\mu \in \mathscr{L}$, we have $V_{2}(1)>0, V_{2}^{\prime}(1)>0$ and $V_{2}(1+1 / D)<0$. In addition one can easily show that $S(1)>0$ for any $\mu \in \mathscr{L}$. (In fact $S(1)$ is precisely two times the first period constant.)

(a) Let us assume $\mu \in \mathscr{L}_{1}$ first. We claim that then $S^{\prime}(1)<0$, and to show this we proceed as follows. First we note that if $\mu \in \mathscr{L}_{1}$, then $F \in(2,2.4)$. Notice next that $S^{\prime}(1)$ is a second degree polynomial in $D$. By computing lower and upper bounds of its coefficients when $F \in(2,2.4)$, we can assert that if $\mu \in \mathscr{L}_{1}$, then $S^{\prime}(1)<-\left(16 D^{2}+48 D+4\right)$, which is negative for $D<-3$. So the claim is true.

Consider at this point the intervals $I_{11}$ and $I_{12}$ in Figure 5. To be more precise, the endpoints of $I_{11}$ are the smallest zeros of $S$ and $V_{2}$, whereas if the local maximum of $S$ is smaller than the largest zero of $V_{2}$, then $I_{12}$ is the segment that joins them. (We remark that eventually $I_{12}$ may not exist.) Then, due to $V_{2}(1)>0, S(1)>0$ and $S^{\prime}(1)<0$, it is clear that either $1 \in I_{11}$ or $1 \in I_{12}$. To show the result we must rule out the second possibility. Due to $V_{2}^{\prime}(1)>0$, if $1 \in I_{12}$, then the local maximum of $V_{2}$ should be larger than that of $S$. However this is not possible because one can easily show that the zero of $V_{2}^{\prime}$ is in between the two zeros of $S^{\prime}$. Hence $1 \in I_{11}$. Finally $z=1+\frac{1}{D}$ is located as shown in Figure 4 because $D$ and $V_{2}\left(1+\frac{1}{D}\right)$ are negative.

(b) The result for $\mu \in \mathscr{L}_{2}$ is obvious using that $V_{2}(1)>0$ and $S(1)>0$.

(c) Assume finally that $\mu \in \mathscr{L}_{3}$, and let us show first that then $S^{\prime}(1)>0$ and $S^{\prime \prime}(1)<0$. To see this we shall use that if $\mu \in \mathscr{L}_{3}$, then $F \in(1.29,1.34)$. We prove that $S^{\prime}(1)>0$ first. As before we consider $S^{\prime}(1)$ as a polynomial in $D$ and, by computing the lower and upper bounds of its coefficients for $F \in(1.29,1.34)$, we can assert that if $\mu \in \mathscr{L}_{3}$, then $S^{\prime}(1)>10 D^{2}+15 D-0.24$, which is positive for $D<-3$. Hence $S^{\prime}(1)>0$ for $\mu \in \mathscr{L}_{3}$. Let us show $S^{\prime \prime}(1)<0$ next. To this end it suffices to check that $h(\mu):=2(16 F-19) D+(9 F-5)(F-1)<0$. The coefficient of $D$ in $h(\mu)$ is positive for $F \in(1.29,1.34)$. Accordingly, if $(D, F) \in \mathscr{L}_{3}$ then $h(D, F)<h(-3, F)=9 F^{2}-110 F+119$, which is negative for $F \in(1.29,1.34)$. Therefore $S^{\prime \prime}(1)<0$ for $\mu \in \mathscr{L}_{3}$.

Now, see Figure 4 , let $I_{21}$ be the segment that joins the largest zero of $V_{2}$ and the second zero of $S$, and let $I_{22}$ be the unbounded interval from the largest zero of $S$ to $+\infty$. Using that $S(1), V_{2}(1)$ and $S^{\prime}(1)$ are positive, we get that either $1 \in I_{21}$ or $1 \in I_{22}$. However $1 \notin I_{22}$ because otherwise $S^{\prime \prime}(1)>0$ and this is not possible for $\mu \in \mathscr{L}_{3}$ as we showed previously. It only remains to place $z=1+\frac{1}{D}$ but this is easy using that $D$ and $V_{2}(1+1 / D)$ are negative.

This concludes the proof of the result.

Lemma 3.5. If $\mu \in \mathscr{L}$, then $S\left(1-\frac{1}{D}\right)>0$.

Proof. A computation shows that $S\left(1-\frac{1}{D}\right)=\frac{2}{D} \ell(\mu)$ with

$$
\ell(\mu):=10 D^{3}+3(10 F-11) D^{2}+3(4 F-3)(3 F-5) D+9 F^{3}-36 F^{2}+48 F-22 .
$$


So it suffices to prove that $\ell(\mu)>0$ for all $\mu \in \mathscr{L}$. One can check that if $\mu \in \mathscr{L}$, then $F \in(1.29,2.32)$. Note on the other hand that $\ell(\mu)$ is a third degree polynomial in $D$. By computing lower and upper bounds of its coefficients for $F \in(1.29,2.32)$, we can assert that $\ell(\mu)<10 D^{3}+37 D^{2}-7.4 D+8$, which is negative for $D<-4$. This proves the result.

Proof of Proposition 3.2. Lemma 2.4 in [14] shows that condition $I_{2}$ of Schaaf's criterium is verified for $\mu \in \mathscr{L}$. Hence we must only study $I_{1}$. To this end (see Remark 2.4) we rewrite the functions that appear in Schaaf's criterium in terms of $z=g(u)$, where $g(u)=(F u+1)^{\frac{-1}{F}}$. By Lemma 3.3, $g(\mathcal{I}) \subset\left(1+\frac{1}{D}, 1-\frac{1}{D}\right)$, and so to verify that $I_{1}$ holds it suffices to show that if $\mu \in \mathscr{L}$, then

$$
S(z)>0 \text { for any } z \in\left(1+\frac{1}{D}, 1-\frac{1}{D}\right) \text {. }
$$

Let us consider the case $F \notin\{2,4 / 3\}$ first. We can then apply Lemma 3.4, which enables us to locate $z=1+\frac{1}{D}$ and $z=1$ among the zeros of $S$ and $V_{2}$. Since $z^{\star}:=1-\frac{1}{D}$ is on the right of $z=1$ due to $D<0$, the assertion in (11) will follow if $z^{\star}$ is placed "correctly" with respect to the zeros of $S$. To make this precise let us denote the three zeros of $S$ by $s_{1}, s_{2}$ and $s_{3}$ taking $s_{1}<s_{2}<s_{3}$. We claim, see Figure 4 , that $z^{\star}<s_{1}$ for $\mu \in \mathscr{L}_{1}, z^{\star} \in\left(s_{2}, s_{3}\right)$ for $\mu \in \mathscr{L}_{2}$ and $z^{\star} \in\left(s_{1}, s_{2}\right)$ for $\mu \in \mathscr{L}_{3}$. Clearly if the claim is true, then (11) follows. The location of $z^{\star}$ among the zeros of $S$ can only change at those $\mu \in \mathscr{L}$ for which either $S\left(z^{\star}\right)=0$ or the leading coefficient of $S$ or its discriminant vanishes. From Lemma 3.5 we know that $S\left(z^{\star}\right) \neq 0$ for any $\mu \in \mathscr{L}$. The leading coefficient of $S$, see Remark 2.4, vanishes at $D(3 F-4)(F-2)=0$. On the other hand a computation shows that its discriminant vanishes at $D(F-2) \kappa(\mu) R(\mu)=0$, where

$$
\kappa(\mu)=(F-1)^{2}+(2 D+1)^{2}-1,
$$

and $R$ is given in (2). Since these curves do not cut any $\mathscr{L}_{i}$, the location of $z^{\star}$ among the zeros of $S$ remains unchanged inside each one of these regions. (Here we use that $\mathscr{L}_{1}, \mathscr{L}_{2}$ and $\mathscr{L}_{3}$ are connected.) Therefore it suffices to verify the claim for one concrete parameter in each $\mathscr{L}_{i}$. We did so, but it is not included here for the sake of shortness. This proves the claim, and so the result follows for $\mu \in \mathscr{L}$ with $F \notin\{2,4 / 3\}$.

Let us study next the case $F=4 / 3$, which is the common boundary between the regions $\mathscr{L}_{2}$ and $\mathscr{L}_{3}$. From the previous discussion, the only bifurcation that undergoes the graphics in Figure 4 at $F=4 / 3$ is due to the vanishing of the leading coefficient of $S$. In other words, $S$ "loses" one of its zeros to become a second degree polynomial. The coefficient of $z^{2}$ at $F=4 / 3$ is negative for $D<-1 / 2$. Thus, see Figure 4 , reasoning for instance in the graphic corresponding to $\mathscr{L}_{3}$, what happens as $F \nearrow 4 / 3$ is that the largest zero of $S$ goes to $+\infty$. The resulting graphic shows that (11) holds for $F=4 / 3$ as well. This proves the result for $\mu \in \mathscr{L}$ with $F=4 / 3$. Finally the monotonicity for $\mu \in \mathscr{L}$ with $F=2$ follows from the results of Y. Zhao in [18]. (In that paper the author describes completely the behaviour of the period function for the entire straight line $F=2$.)

\subsection{Monotonicity in the straight lines.}

Proposition 3.6. The period function of the center at the origin of system (1) satisfies the following: 


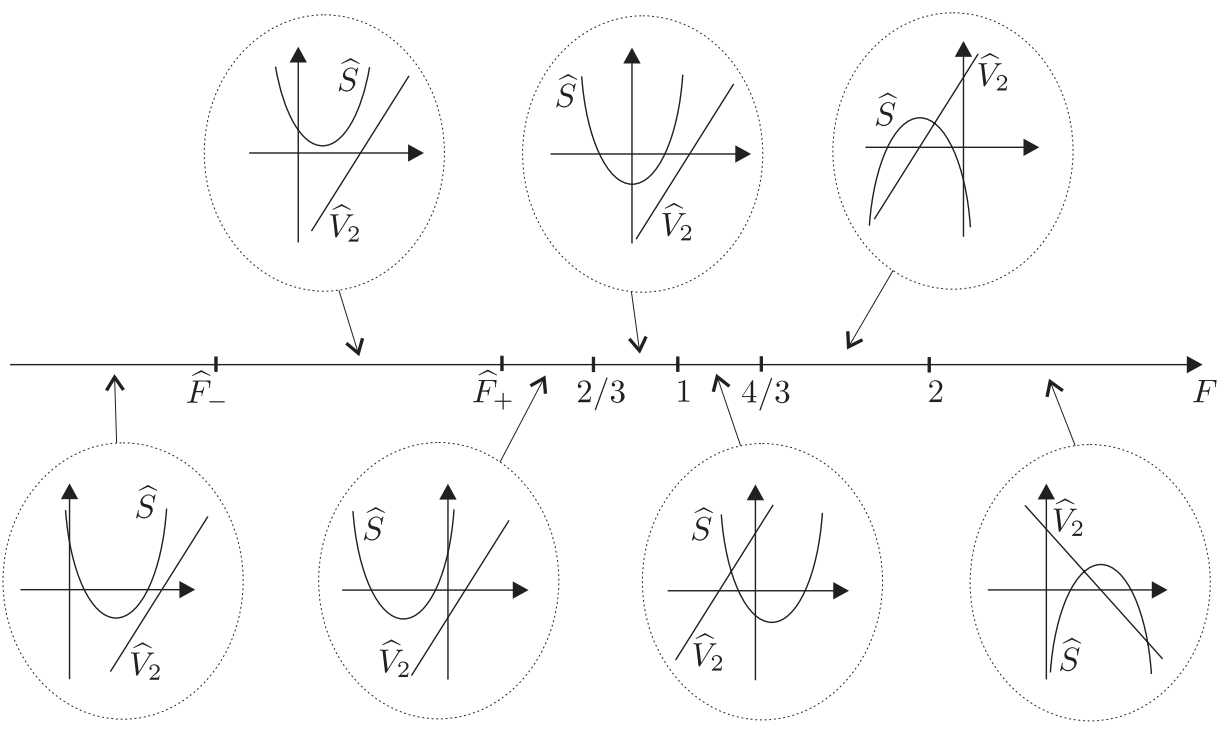

Figure 6. Distribution of the zeros of $\widehat{S}$ and $\widehat{V}_{2}$.

(a) If $F=0$, then the period function is monotonically increasing.

(b) If $D=0$ and $F \notin[0,1]$, then the period function is monotonically increasing. If $D=0$ and $F \in\left(\frac{2}{3}, 1\right)$, then it is monotonically decreasing.

(c) If $D=-1$ and $F \notin(1,3)$, then the period function is monotonically increasing. If $D=-1$ and $F \in\left(\frac{4}{3}, 2\right)$, then it is monotonically decreasing.

(d) If $F=2$ and $D \notin(-2,0)$, then the period function is monotonically increasing.

Proof. The proofs of $(a)$ and $(b)$ are omitted for the sake of brevity because they follow in a straightforward manner by applying Schaaf's criterium. The statement in $(d)$ is proved in [18].

As usual, to show $(c)$ we follow the approach explained in Remark 2.4. The verification of Schaaf's conditions is easier than the general case because $S$ and $V_{2}$ factor. More concretely one can check that $V_{2}(z)=z \widehat{V}_{2}(z)$ and $S(z)=z \widehat{S}(z)$, with $\widehat{V}_{2}(z):=F-1-(F-2) z$ and

$$
\widehat{S}(z):=-2(3 F-4)(F-2)^{2} z^{2}+(9 F-5)(F-1)(F-2) z-(3 F-2)(F-1)^{2} .
$$

Since $z=g(u)>0$, condition $I_{1}$ is equivalent to showing that

$$
\widehat{S}(z)>0 \text { for any } z \in g(\mathcal{I}) \text { with } \widehat{V}_{2}(z)>0 .
$$

Note that $\widehat{V}_{2}$ vanishes at $\bar{z}:=\frac{F-1}{F-2}$. Some computations show that $V_{1}(\bar{z}) V_{3}(\bar{z})=-\bar{z}^{3}$, and this proves that $I_{2}$ holds because any $z=g(u)$ must be positive. Figure 6 shows the distribution of the zeros of $\widehat{S}$ and $\widehat{V}_{2}$ and their location with respect to $z=0$ in terms of $F$. (In this figure $\widehat{F}_{ \pm}=-3 \pm \frac{2}{3} \sqrt{30}$ are zeros of the discriminant of $S$.) In view of these graphics it follows that conditions $I_{1}$ and $\mathscr{D}$ are satisfied for $F<1$ and $F \in\left(\frac{4}{3}, 2\right)$ respectively. Our next goal is to show that $I_{1}$ is satisfied for $F>3$ as well. To this end we shall use that if $D=-1$, then

$$
\mathcal{I}=\left(-\frac{1}{F}, \frac{\left(\frac{2 F-2}{2 F-1}\right)^{-F}-1}{F}\right) \text {. }
$$


(This follows easily by taking the expression of $V$ in (6) into account.) Hence it turns out that $g(\mathcal{I})=\left(z_{0},+\infty\right)$, where $z_{0}:=\frac{2 F-2}{2 F-1}$. Accordingly, in view of the graphic corresponding to $F>2$, condition $I_{1}$ is verified for those $F$ such that $\widehat{S}\left(z_{0}\right)>0$. One can check that

$$
\widehat{S}\left(z_{0}\right)=\frac{(F-1)^{2}\left(38 F^{2}-149 F+110\right)}{(2 F-1)^{2}},
$$

which is positive for $F>\frac{149+3 \sqrt{609}}{76} \approx 2.93$. This concludes the proof of $(c)$.

\section{REFERENCES}

[1] Changjian Liu, The monotonicity of the periods in some class of reversible quadratic centers, preprint (2006).

[2] C. Chicone, review in MathSciNet, ref. 94h:58072.

[3] R. Chouikha, Monotonicity of the period function for some planar differential systems. I. Conservative and quadratic systems, Appl. Math. 32 (2005), 305-325. MR2213617 (2007d:34079)

[4] W.A. Coppel and L. Gavrilov, The period function of a Hamiltonian quadratic system, Differential Integral Equations 6 (1993), 1357-1365. MR1235199 (94h:58072)

[5] A. Gasull, A. Guillamon and J. Villadelprat, The period function for second-order quadratic ODEs is monotone, Qual. Theory Dyn. Syst. 5 (2004), 201-224. MR2129724 (2005k:34115)

[6] P. Mardešić, D. Marín and J. Villadelprat, On the time function of the Dulac map for fmailies of meromorphic vector fields, Nonlinearity 16 (2003), 855-881. MR1975786 (2004k:37031)

[7] P. Mardešić, D. Marín and J. Villadelprat, The period function of reversible quadratic centers, J. Differential Equations 224 (2006), 120-171. MR2220066 (2006m:34076)

[8] D. Marín and J. Villadelprat, On the return time function around monodromic polycycles, J. Differential Equations 228 (2006), 226-258. MR2254430 (2007d:34061)

[9] F. Rothe, The periods of the Volterra-Lokta system, J. Reine Angew. Math. 355 (1985), 129-138. MR772486 (86c:92026)

[10] F. Rothe, Remarks on periods of planar Hamiltonian systems, SIAM J. Math. Anal. 24 (1993), 129-154. MR1199531 (93m:34058)

[11] R. Schaaf, Global behaviour of solution branches for some Neumann problems depending on one or several parameters, J. Reine Angew. Math. 346 (1984), 1-31. MR727393 (85i:58035)

[12] R. Schaaf, A class of Hamiltonian systems with increasing periods, J. Reine Angew. Math. 363 (1985), 96-109. MR814016 (87b:58029)

[13] J. Villadelprat, The period function of the generalized Lotka-Volterra centers, preprint (2006).

[14] J. Villadelprat, On the reversible quadratic centers with monotonic period function, Proc. Amer. Math. Soc. 135 (207), 2555-2565. MR2302576

[15] J. Waldvogel, The period in the Lotka-Volterra system is monotonic, J. Math. Anal. Appl. 114 (1986), 178-184. MR829122 (87j:92034)

[16] Y. Zhao, The monotonicity of period function for codimension four quadratic system $Q_{4}$, J. Differential Equations 185 (2002), 370-387. MR1938124 (2003g:34057)

[17] Y. Zhao, The period function for quadratic integrable systems with cubic orbits, J. Math. Anal. Appl. 301 (2005), 295-312. MR2105672 (2005h:34082)

[18] Y. Zhao, On the monotonicity of the period function of a quadratic system, Discrete Contin. Dyn. Syst. 13 (2005), 795-810. MR2153144 (2006d:34072)

Departament de Matemàtiques, Universitat Autònoma de Barcelona, 08193 Bellaterra, Barcelona, Spain

Departament D'Enginyeria Informàtica i MatemÀtiques, Universitat Rovira i Virgili, 43007 TARragona, Spain 